\title{
Diverse bacterial communities exist on canine skin and are impacted by cohabitation and time
}

\author{
Sheila Torres ${ }^{1}$, Jonathan B Clayton ${ }^{2}$, Jessica L Danzeisen ${ }^{2}$, Tonya Ward ${ }^{3}$, Hu Huang ${ }^{3}$, Dan Knights ${ }^{3,4}$, \\ Timothy J Johnson Corresp. 2 \\ 1 Department of Veterinary Clinical Sciences, University of Minnesota, Saint Paul, Minnesota, United States \\ 2 Department of Veterinary and Biomedical Sciences, University of Minnesota, Saint Paul, Minnesota, United States \\ 3 Biotechnology Institute, University of Minnesota, Minneapolis, Minnesota, United States \\ 4 Department of Computer Science and Engineering, University of Minnesota, Minneapolis, Minnesota, United States \\ Corresponding Author: Timothy J Johnson \\ Email address: joh04207@umn.edu
}

It has previously been shown that domestic dogs and their same-household owners share bacterial populations, and that sharing of bacteria between humans is facilitated through the presence of dogs in the household. However, less is known regarding the bacterial communities of dogs, how these communities vary by location and over time, and how cohabitation of dogs themselves influences their bacterial community. Furthermore, the effects of factors such as breed, hair coat length, sex, shedding, and age on the canine skin microbiome is unknown. This study sampled the skin bacterial communities of $\mathbf{4 0}$ dogs belonging to 20 households longitudinally across three seasons (Spring, Summer, and Winter). Significant differences in bacterial community structure between samples were identified when stratified by season, but not by dog sex, age, breed, hair type, or skin site. Cohabitating dogs were more likely to share bacteria of the skin than non-cohabitating dogs. Similar to human bacterial microbiomes, dogs' microbiomes were more similar to their own microbiomes over time than to microbiomes of other individuals. Dogs sampled during the same season were also more similar to each other than to dogs from different seasons, irrespective of household. However, there were very few core operational taxonomic units identified across all dogs sampled. Taxonomic classification revealed Propionibacterium acnes and Haemophilus sp. as key members of the dog skin bacterial community, along with Corynebacterium sp. and Staphylococcus epidermidis. This study shows that the skin bacterial community structure of dogs is highly individualized, but can be shared among dogs through cohabitation. 
1 Diverse bacterial communities exist on canine skin and are impacted by

2 cohabitation and time

3

4

5

6

7

8

9
Sheila Torres ${ }^{1}$, Jonathan B. Clayton ${ }^{2}$, Jessica L. Danzeisen ${ }^{2}$, Tonya Ward ${ }^{3}$, Hu Huang ${ }^{3}$, Dan Knights $^{3,4}$, and Timothy J. Johnson ${ }^{2 *}$

${ }^{1}$ University of Minnesota, Department of Veterinary Clinical Sciences, Saint Paul, MN, USA ${ }^{2}$ University of Minnesota, Department of Veterinary and Biomedical Sciences, Saint Paul, Minnesota, USA

${ }^{3}$ Biotechnology Institute, University of Minnesota, Minneapolis, Minnesota 55455, USA ${ }^{4}$ Department of Computer Science and Engineering, University of Minnesota, Minneapolis, Minnesota 55455, USA

\section{*Corresponding author:}

Timothy Johnson

joh04207@umn.edu 


\section{Abstract}

34 It has previously been shown that domestic dogs and their same-household owners share

35 bacterial populations, and that sharing of bacteria between humans is facilitated through the

36 presence of dogs in the household. However, less is known regarding the bacterial communities

37 of dogs, how these communities vary by location and over time, and how cohabitation of dogs

38 themselves influences their bacterial community. Furthermore, the effects of factors such as

39 breed, hair coat length, sex, shedding, and age on the canine skin microbiome is unknown. This

40 study sampled the skin bacterial communities of 40 dogs belonging to 20 households

41 longitudinally across three seasons (Spring, Summer, and Winter). Significant differences in

42 bacterial community structure between samples were identified when stratified by season, but

43 not by dog sex, age, breed, hair type, or skin site. Cohabitating dogs were more likely to share

44 bacteria of the skin than non-cohabitating dogs. Similar to human bacterial microbiomes, dogs'

45 microbiomes were more similar to their own microbiomes over time than to microbiomes of

46 other individuals. Dogs sampled during the same season were also more similar to each other

47 than to dogs from different seasons, irrespective of household. However, there were very few

48 core operational taxonomic units identified across all dogs sampled. Taxonomic classification

49 revealed Propionibacterium acnes and Haemophilus sp. as key members of the dog skin

50 bacterial community, along with Corynebacterium sp. and Staphylococcus epidermidis. This

51 study shows that the skin bacterial community structure of dogs is highly individualized, but can

52 be shared among dogs through cohabitation.

53

54 


\section{Introduction}

57 The skin contains a very effective physical, immunological and microbial barrier that protects the

58 body against dehydration and constant environmental insults. The bacterial communities of the

59 skin have been well studied, and computational and laboratory advances in the technology of

60 microbial community profiling have enabled more accurate investigation of these communities

61 commonly referred to as the microbiota or microbiome. Various studies using next generation

62 sequencing techniques have shown that the skin bacterial community of healthy humans is quite

63 diverse and its composition, biomass, and diversity are highly influenced by the physiological

64 characteristics of the cutaneous microenvironment (Costello et al. 2009; Grice et al. 2009; Grice

65 et al. 2008; Grice \& Segre 2011). Additional studies have shown that age and environmental

66 factors such as cohabitation and having a dog also influence the composition and diversity of the

67 skin bacterial community of healthy humans (Capone et al. 2011; Dominguez-Bello et al. 2010;

68 Oh et al. 2012; Song et al. 2013). Moreover, the temporal stability of the healthy human skin

69 microbiome was recently investigated and diversity, skin site and individuality were all

70 determinants of stability (Flores et al. 2014; Oh et al. 2016).

71

72 Despite the wealth of information regarding the skin microbiome of healthy humans, current

73 knowledge in healthy domestic dogs (Canis familiaris) comes primarily from a study by

74 Hoffmann et al (Rodrigues Hoffmann et al. 2014). This study showed that the canine bacterial

75 community is diverse and quite variable across different body sites within the same dog, and

76 across the same site in different dogs, suggesting that the skin microenvironment in dogs does

77 not substantially impact the composition of its bacterial community. Similar to human skin, the

78 most abundant phyla identified in dogs were Proteobacteria, Firmicutes, Actinobacteria and 
79 Bacteriodetes; respectively, however, Actinobacteria has been shown to predominate in humans

80 whereas it was less abundant in dogs (Costello et al. 2009; Grice et al. 2009; Grice et al. 2008;

81 Grice \& Segre 2011; Rodrigues Hoffmann et al. 2014). Host and environmental factors such as

82 age, sex, breed, fleas and time spent outside do not appear to influence the composition of the

83 bacterial community in dogs. The study by Hoffmann et al has improved our knowledge on the

84 composition of the skin microbiome of healthy dogs, previously based only on culture-dependent

85 methods. However, there is still much to be learned regarding the structure of the microbial

86 communities that live on the skin of healthy dogs and the factors that shape these communities.

87

88 The primary aims of this study were to evaluate if there is a core bacterial community living on 89 the skin of healthy domestic dogs from Minnesota, USA, and if body site, dog cohabitation and 90 seasonality have an impact on this community.

91

92 Methods

93 Study design

94 Healthy, privately-owned paired $\operatorname{dogs}(\mathrm{n}=40)$ of various breeds from 20 households were

95 enrolled in this study through the University of Minnesota Veterinary Medical Center. Dogs

96 belonged to local clients or employees living in proximity to the Twin Cities, Minnesota, USA.

97 Owners signed an informed consent at the time of enrollment and were allowed to withdraw their

98 dogs at any time during the study. To be included in the study the dogs were required to 1) be

99 healthy based on a thorough history and clinical signs; 2) not receive any systemic or topical

100 antimicrobial therapy for at least 3 months prior to enrollment; and 3) not be bathed for at least

10130 days before inclusion. Furthermore, in order for a household to participate in the study, none 
102 of the animals in the household could have skin or ear disease, and cohabiting dogs had to be

103 living together for at least 6 months and spend at least $80 \%$ of the time together. The subjects

104 consisted of 13 females and 27 males, with an average age of 7.6 years (Table 1). All animal

105 work was carried out in accordance with the Institutional Animal Care and Use Committee at the

106 University of Minnesota, protocol number 1108A03922.

107

108 Table 1. Summary of enrolled dogs in this study.

\begin{tabular}{|c|c|c|c|c|}
\hline Dog & Breed & Age & Gender ${ }^{\mathrm{a}}$ & Coat \\
\hline $1 \mathrm{~A}$ & Dachshund & 12 & FS & Long/medium \\
\hline 1B & Dachshund & 7 & $\mathrm{MN}$ & Long/medium \\
\hline $2 \mathrm{~A}$ & Siberian Husky & 7 & $\mathrm{MN}$ & Long/medium \\
\hline $2 \mathrm{~B}$ & Mixed & 11 & FS & Long/medium \\
\hline $3 \mathrm{~A}$ & Labrador & 7 & $\mathrm{MN}$ & Long/medium \\
\hline $3 \mathrm{~B}$ & Yorkshire Terrier & 2 & $\mathrm{MN}$ & Long/medium \\
\hline $4 \mathrm{~A}$ & Chihuahua & 5 & $\mathrm{MN}$ & Short \\
\hline $4 \mathrm{~B}$ & Greyhound & 8 & FS & Short \\
\hline $5 \mathrm{~A}$ & Mixed & 11 & $\mathrm{MN}$ & Long/medium \\
\hline $5 B$ & Italian Greyhound & 9 & $\mathrm{MN}$ & Short \\
\hline $6 \mathrm{~A}$ & Boston Terrier & 12 & $\mathrm{MN}$ & Short \\
\hline $6 \mathrm{~B}$ & Boston Terrier & 13 & FS & Short \\
\hline $7 \mathrm{~A}$ & Newfoundland & 10 & $\mathrm{MN}$ & Long \\
\hline $7 \mathrm{~B}$ & Jack Russell Terrier & 3 & $\mathrm{MN}$ & Short \\
\hline $8 \mathrm{~A}$ & Dachshund & 3 & $\mathrm{MN}$ & Medium/wire \\
\hline
\end{tabular}




\begin{tabular}{|c|c|c|c|c|}
\hline $8 B$ & Dachshund & 3 & $\mathrm{MN}$ & Medium/wire \\
\hline $9 \mathrm{~A}$ & Mixed & 10 & $\mathrm{MN}$ & Long/medium \\
\hline $9 \mathrm{~B}$ & Greyhound & 9 & $\mathrm{MN}$ & Short \\
\hline $10 \mathrm{~A}$ & Miniature Poodle & 14 & $\mathrm{MN}$ & Short \\
\hline $10 \mathrm{~B}$ & Miniature Poodle & 6 & $\mathrm{MN}$ & Short \\
\hline $11 \mathrm{~A}$ & Malamute & 5 & FS & Long/medium \\
\hline $11 B$ & Siberian Husky & 6 & $\mathrm{MN}$ & Long/medium \\
\hline $12 \mathrm{~A}$ & Mixed & 4 & $\mathrm{MN}$ & Long/medium \\
\hline $12 \mathrm{~B}$ & Mixed & 8 & $\mathrm{MN}$ & Long/medium \\
\hline $13 \mathrm{~A}$ & Mixed & 10 & FS & Short \\
\hline $13 \mathrm{~B}$ & Mixed & 4 & FS & Short \\
\hline $14 \mathrm{~A}$ & Great Dane & 7 & FS & Short \\
\hline $14 \mathrm{~B}$ & Cavalier Spaniel & 2 & $\mathrm{MN}$ & Long/medium \\
\hline $15 \mathrm{~A}$ & Shih Tzu & 4 & FS & Long/medium \\
\hline $15 B$ & Shih Tzu & 4 & $\mathrm{MN}$ & Long/medium \\
\hline $16 \mathrm{~A}$ & Malamute & 6 & FS & Long/medium \\
\hline $16 \mathrm{~B}$ & Mixed & 4 & $\mathrm{MN}$ & Long/medium \\
\hline $17 \mathrm{~A}$ & Samoyed & 9 & MI & Long \\
\hline 17B & Australian Shepherd & 14 & $\mathrm{MN}$ & Long/medium \\
\hline $18 \mathrm{~A}$ & Mixed & 10 & $\mathrm{MN}$ & Long/medium \\
\hline $18 \mathrm{~B}$ & Mixed & 10 & $\mathrm{MN}$ & Long/medium \\
\hline $19 \mathrm{~A}$ & Chihuahua & 5 & $\mathrm{MN}$ & Short \\
\hline 19B & Chihuahua & 14 & FS & Short \\
\hline
\end{tabular}




\begin{tabular}{lllll}
\hline 20A & Siberian Husky & 10 & FS & Long/medium \\
20B & Siberian Husky & 6.5 & FI & Long/medium
\end{tabular}

${ }^{\mathrm{a} F S}$, female spayed; MN, male neutered; MI, male intact; FI, female intact.

110

111 At three timepoints spaced approximately three months apart (designated winter, spring and

112 summer), samples were collected from three sites on each dog (dorsal neck, axilla, and

113 abdomen). Skin samples were collected by shaving a $10 \mathrm{~cm}^{2}$ area at each site, and swabbing 25

114 times with a nylon-flocked swab (Copan Diagnostic Inc, Murrieta, CA) moistened in SCF-1

115 (50mM Tris, $\mathrm{pH} 7.6,1 \mathrm{mM}$ EDTA, 0.5\% Tween-20). All samples were stored at $4^{\circ} \mathrm{C}$ and

116 processed within 2 hours without freezing.

\section{Sample Processing and sequencing.}

119 DNA was extracted using Mo Bio UltraClean®-htp 96 Well Microbial DNA kit (Mo Bio

120 Laboratories, Carlsbad, CA), according to the manufacturer's directions. Amplification of the

121 16S rRNA gene was performed using KAPA HiFidelity Hot Start Polymerase (Kapa Biosystems,

122 Inc., Wilmington, MA) for two rounds of PCR at the University of Minnesota Genomics Center

123 (Minneapolis, MN). For the first round, the V1V3F (5'-

124 GTCTCGTGGGCTCGGAGATGTGTATAAGAGACAGAGAGTTTGATCMTGGCTCAG -

125 3') and V1V3R (5'-

\section{TCGTCGGCAGCGTCAGATGTGTATAAGAGACAGATTACCGCGGCTGCTGG -3’)}

127 Nextera primers (Integrated DNA Technologies, Coralville, IA) were used to amplify the V1-V3

128 hypervariable region using the following cycling parameters: one cycle of $95^{\circ} \mathrm{C}$ for five minutes, 129 followed by 20 cycles of $98^{\circ} \mathrm{C}$ for 20 seconds, $55^{\circ} \mathrm{C}$ for 15 seconds, and $72^{\circ} \mathrm{C}$ for one minute. 
130 The products were then diluted 1:100 and 5ul was used in a second round of PCR using forward

131 (5' - CAAGCAGAAGACGGCATACGAGAT[i5]GTCTCGTGGGCTCGG -3') and reverse

132 (5' - AATGATACGGCGACCACCGAGATCTACAC[i7]TCGTCGGCAGCGTC -3') indexing

133 primers (Integrated DNA Technologies). The second PCR used the following cycling

134 parameters: 1 cycle at $95^{\circ} \mathrm{C}$ for 5 minutes, followed by 10 cycles of $98^{\circ} \mathrm{C}$ for 20 seconds, $55^{\circ} \mathrm{C}$

135 for 15 seconds, and $72^{\circ} \mathrm{C}$ for 1 minute. Pooled, size-selected samples were denatured with

$136 \mathrm{NaOH}$, diluted to $8 \mathrm{pM}$ in Illumina's HT1 buffer, spiked with 15\% PhiX, and heat denatured at

$13796^{\circ} \mathrm{C}$ for 2 minutes immediately prior to loading. A MiSeq 600 (2X300 bp) cycle v3 kit

138 (Illumina, San Diego, CA) was used to sequence the samples.

139

140 Data analyses.

141 Following sequencing, sorting by barcode was performed to generate fastq files for each sample.

142 Proximal and distal primers were trimmed from the sequence reads. Open referenced operational

143 taxonomic unit (OTU) picking was used in QIIME (Caporaso et al. 2010) using uclust (Edgar

144 2010). Potential chimeras were removed using ChimeraSlayer (Haas et al. 2011). OTUs present

145 in negative control amplifications were also removed prior to subsequent analysis. After filtering

146 due to low yield on some samples, a total of 40 abdomen, 46 dorsal neck, and 52 axilla samples

147 ( $\mathrm{n}=138)$ were retained for analysis following sequencing, quality filtering, and OTU clustering at

$14897 \%$ sequence similarity. Samples were rarefied to 5,000 high quality reads per sample.

149

150 QIIME was used for assessments of alpha diversity, beta diversity using Unifrac (Lozupone \&

151 Knight 2005), phylogenetic classifications using the Greengenes database (Cole et al. 2009), and

152 core bacterial community structure. Statistical analyses for differences in taxa between body site 
153 and season were performed using the Kruskal-Wallis test with correction for false discovery rate

154 at 0.05 . Statistical differences in overall community structure were performed in R using distance

155 matrices analyzed via the ANOSIM command in QIIME (for beta diversity) and a nonparametric

156 two sample t-test (for alpha diversity).

157

158 The raw data from this project is freely available at the Data Repository for the University of

159 Minnesota (DRUM) at the following link: http://doi.org/10.13020/D6W01V.

160

161

\section{Results}

163 From 360 total samples, 138 samples were retained following removal of samples due to

164 insufficient DNA for sequencing or low sequencing output (Table 2). Most failures were due to

165 low DNA yield and the stringent conditions used for quality assessment and filtering of

166 sequences. While all samples were subjected to DNA amplification and sequencing, many had

167 fewer than 5,000 total reads which were subsequently discarded. Some of these samples were

168 tested on subsequent runs with the same results. The total number of reads per sample in those

169 used ranged from 5,016 to 297,512. Following filtering of OTUs not classified as bacteria, 6,966

170 OTUs were retained for downstream analyses. All samples were then rarefied to 5,000 sequences

171 for subsequent analysis.

172

173

174

175 
176 Table 2. Number of samples analyzed in this study by body site and season.

\begin{tabular}{|l|r|r|l|r|}
\hline & & Abdomen & Axilla & \multicolumn{2}{l|}{$\begin{array}{l}\text { Dorsal } \\
\text { Neck }\end{array}$} \\
\hline Spring & 15 & 19 & 26 & 60 \\
\hline Summer & 16 & 14 & 7 & 37 \\
\hline Winter & 9 & 19 & 13 & 41 \\
\hline Total & 40 & 52 & 46 & 138 \\
\hline
\end{tabular}

177

178 Samples were first taxonomically classified at the bacterial Class level by QIIME using OTUs

179 and the Greengenes database (Figure 1). When categorized by skin site or season, a range of

180 differences was seen from sample-to-sample within each site, but these ranges did not visually

181 differ between sites. The dominant classes were Actinobacteria (range 0-75.6\%), Bacilli (range

182 0-62.2\%), and Gammaproteobacteria (range 0-56.4\%).

183

184

185

186

187

188

189

190

191

192

193 
194 Figure 1. Individual samples classified by Greengenes according to bacterial class, grouped by

195 skin site, and ordered by sample number. The top chart is Spring samples, Middle chart is

196 Summer samples, and bottom chart is Winter samples.
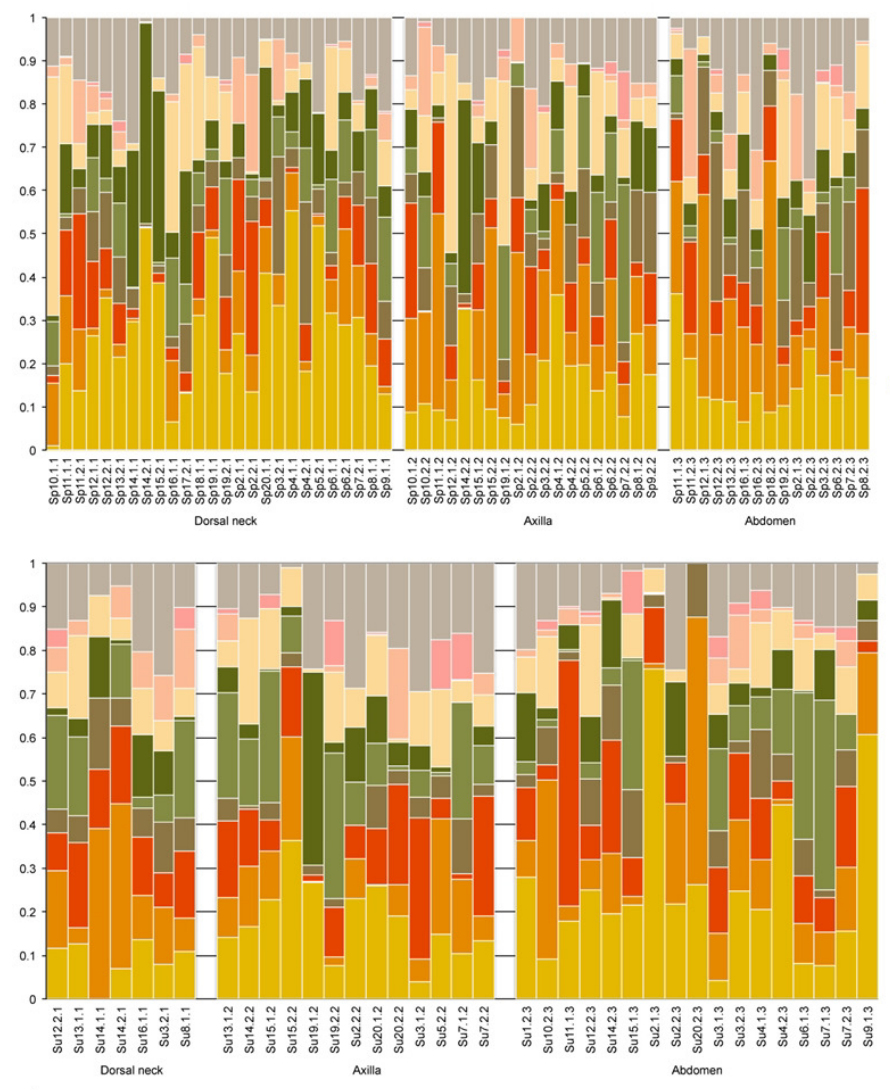

- Other

- Fusobacteriia

- Flavobacteriia

Clostridia

- Alphaproteobacteria

- Bacteroidia

- Betaproteobacteria

- Gammaproteobacteria

- Bacilli

- Actinobacteria

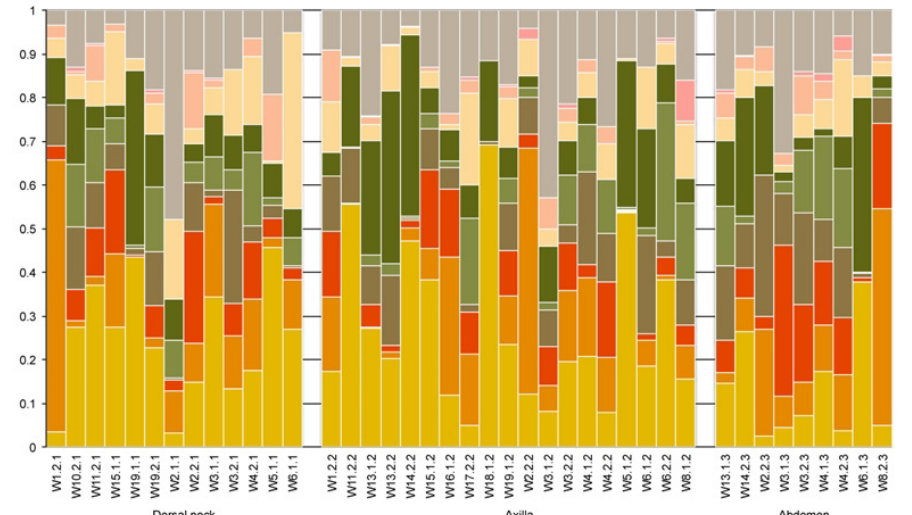

199 OTU-based analyses were then performed using measures of alpha diversity and beta diversity.

200 High Shannon diversity indices were observed across all samples whether grouped by season or 
201 skin site (Figure 2). Using phylogenetic diversity across the entire tree and then Shannon

202 diversity indices, no significant differences in alpha diversity were observed when grouping

203 samples by age, sex, breed, hair type, season, or skin site.

204

205 Figure 2. Beeswarm plots of Shannon diversity values with median and interquartile ranges for 206 samples grouped by season (A) and skin site (B).

207

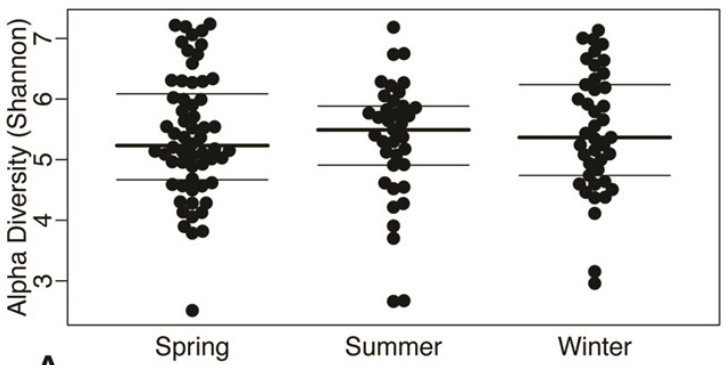

A

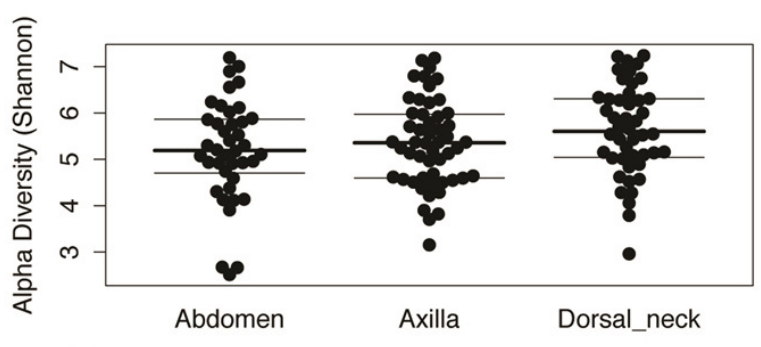

B

208 Beta diversity was compared between samples using principle coordinate analysis (PCoA) plots

209 (Figure 3). There was no visual clustering of samples either by season or skin site. When

210 assessing beta diversity using the anosim function in QIIME, no significant differences were

211 identified when grouping by age, sex, breed, hair type, or skin site. However, significant

212 differences in community composition were identified when grouping by season $(\mathrm{P}=0.003)$.

213 When statistically different taxa at the OTU level were assessed by season, 13 total taxa were

214 identified. Of these, the only differential taxa of appreciable relative abundance were those

215 classified as Actinomycetales (class level) which was of lower relative abundance in the

216 Summer, and Actinomyces (genus) which was also of lower relative abundance in the Summer. 
219 Figure 3. Principle coordinate analysis (PCoA) plots of individual samples. On the left, samples 220 are colored by skin site using unweighted matrices $(\mathrm{A})$ or weighted matrices $(\mathrm{C})$. On the right,

221 samples are colored by season site using unweighted matrices (B) or weighted matrices (D).
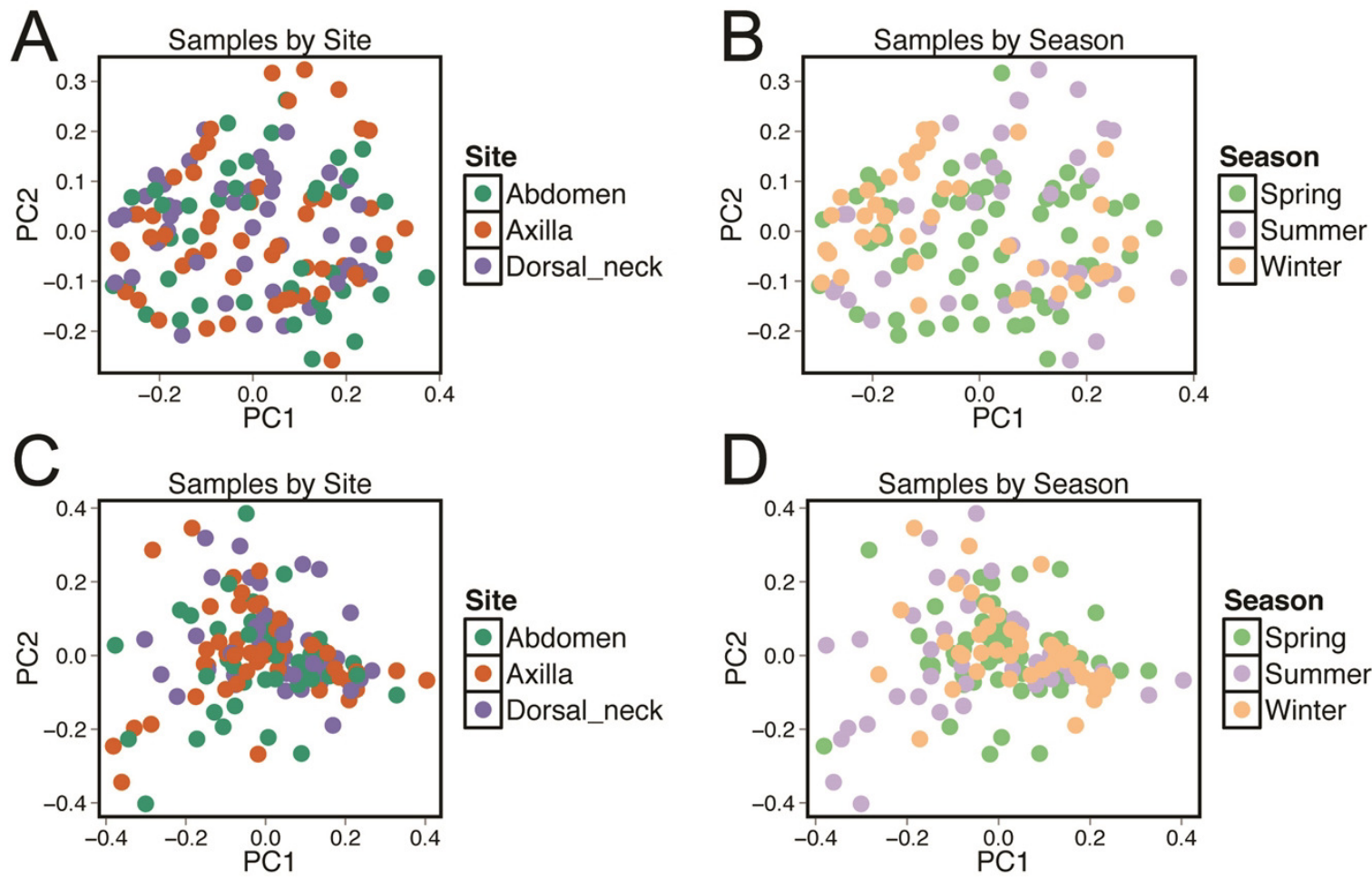

222

223

224 Unifrac distances were used to examine the dissimilarity between samples grouped by a variety

225 of criteria, including sex (male or female), hair type (short or long), breed, age, skin site, season,

226 household, and individual dog (Figure 4). In the analyses, "same" indicates average dissimilarity

227 between samples within the same grouping, whereas "different" indicates average dissimilarity

228 between samples of different groupings. When comparing "same" samples to "different" samples

229 for each category using unweighted Unifrac distances, significant differences in distance were

230 identified when grouped by season $\left(\mathrm{P}=3.5 \times 10^{-8}\right)$, household $\left(\mathrm{P}=1.2 \times 10^{-7}\right)$, breed $(\mathrm{P}=$

231 0.003), and age $\left(\mathrm{P}=1.7 \times 10^{-5}\right)$. The largest differences between "same" and "different" samples 
232 were observed when categorizing by same dogs within the same season (across multiple skin 233 sites) (Figure 4).

234

235 Figure 4. Comparison of unweighted and weighted Unifrac distance matrices between samples 236 when grouped by a variety of different criteria. "Same" indicates average dissimilarity between 237 samples within the same grouping, whereas "different" indicates average dissimilarity between 238 samples between different groupings. Panel A uses unweighted Unifrac distances and Panel B 239 uses weighted Unifrac distances. Those comparisons that are statistically significant $(\mathrm{P}<0.05)$ 240 are indicated with asterisks. Error bars represent 95\% confidence intervals. 

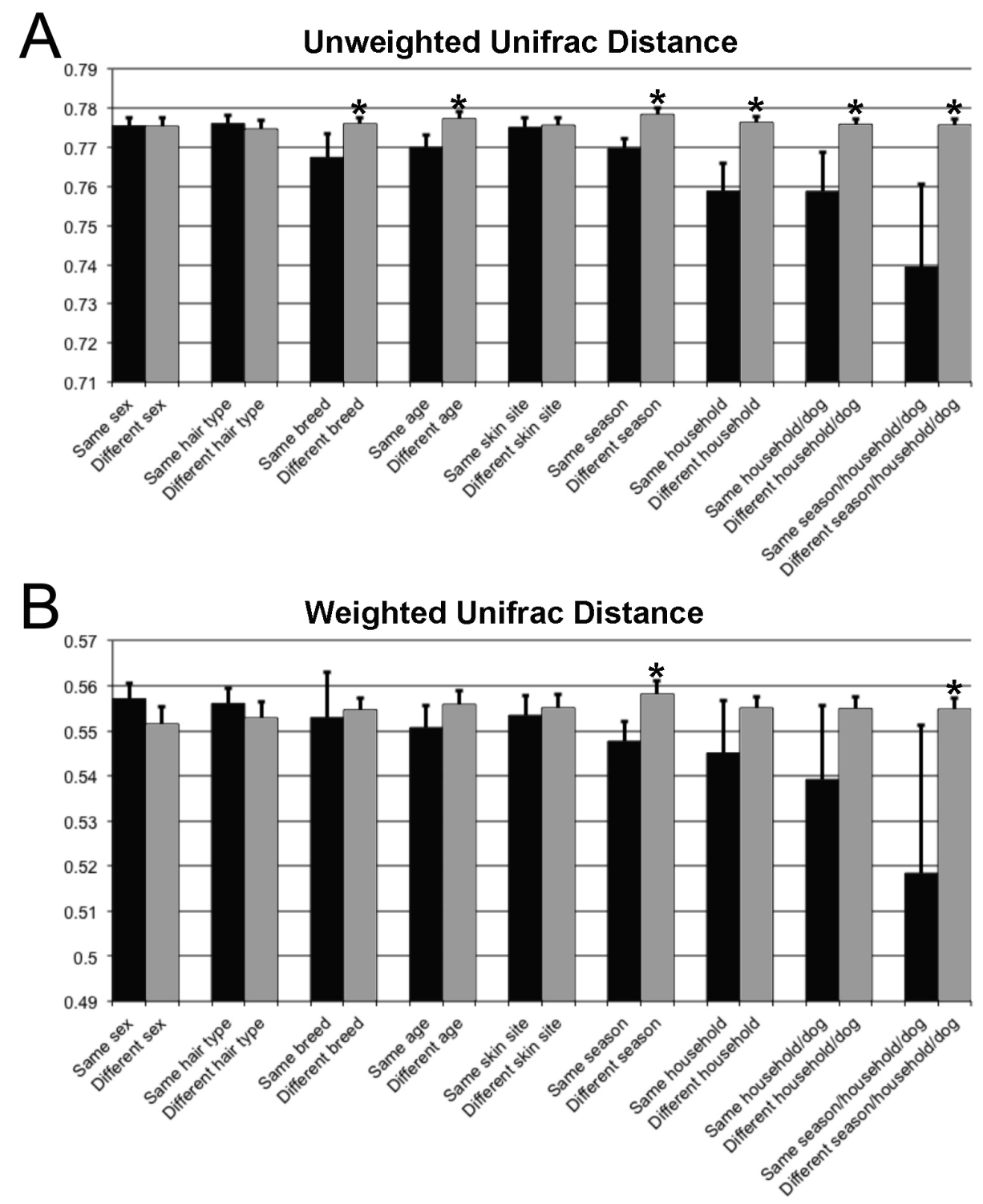

243 The core and dominant OTUs in canine skin were assessed based upon their prevalence amongst

244 samples and their relative abundance. A cutoff of $50 \%$ prevalence across samples was used to

245 assess the core bacterial community because of the lack of any OTUs present in $80 \%$ or greater 246 samples in each group, the established standard for core microbiome definition (Li et al. 2013)

247 (Figure 5). Only two OTUs were identified as core to all groups by season and by skin site. An 248 OTU classified as Propionibacterium acnes was present in $>80 \%,>75 \%$, and $>90 \%$ of Spring, 
249 Summer and Winter samples, respectively, and in $>80 \%,>80 \%$, and $>90 \%$ of abdomen, axilla, 250 and dorsal neck samples, respectively. A second OTU classified as Haemophilus was present in $251>60 \%,>60 \%$, and $>65 \%$ of Spring, Summer and Winter samples, respectively, and in $>60 \%$, $252>55 \%$, and $>70 \%$ of abdomen, axilla, and dorsal neck samples, respectively. An OTU classified 253 as Corynebacterium was dominant in relative abundance across many samples, but highly 254 variable (Figure 6). In particular, it was more prevalent across samples in Winter ( $>95 \%)$ and 255 Spring $(>80 \%)$, and samples from the axilla $(>80 \%)$ and dorsal neck $(>90 \%)$. An OTU classified 256 as Staphylococcus epidermidis was more prevalent amongst abdomen samples (>55\%), and 257 samples from Spring $(>50 \%)$ and Summer $(>55 \%)$.

258

259

260

261

262

263

264

265 Figure 5. OTUs present in $>50 \%$ of all samples by group. Panel A depicts samples by skin site, 266 and Panel B depicts samples by season. 

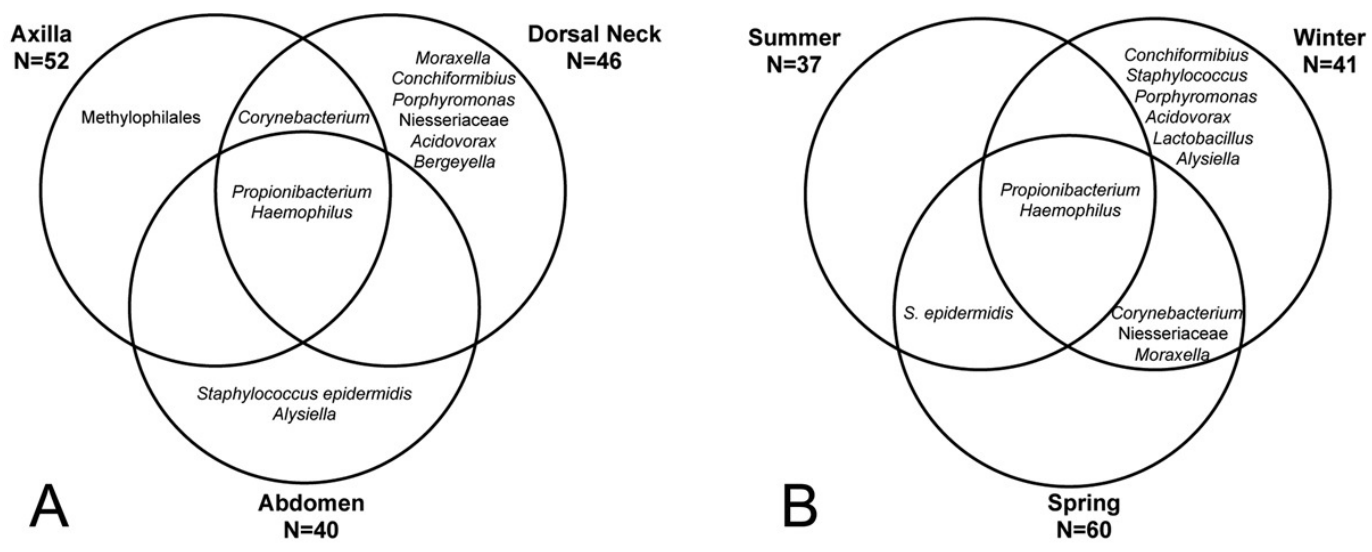

Figure 6. Distribution of selected OTUs identified and classified using the Greengenes database.

270 Beeswarm plots depict individual sample OTU abundance based on 5,000 normalized reads per

271 sample. Boxplots indicate median and quartile ranges for each OTU. The top plot for each OTU

272 categorizes samples by season, whereas the bottom plots categorize samples by skin site. Panel A $273=$ Propionibacterium acnes, Panel $\mathrm{B}=$ Corynebacterium, Panel $\mathrm{C}=$ Haemophilus, and Panel $\mathrm{D}=$ 274 Staphylococcus epidermidis.

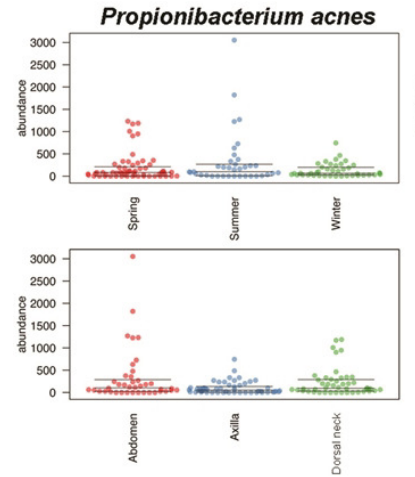

A
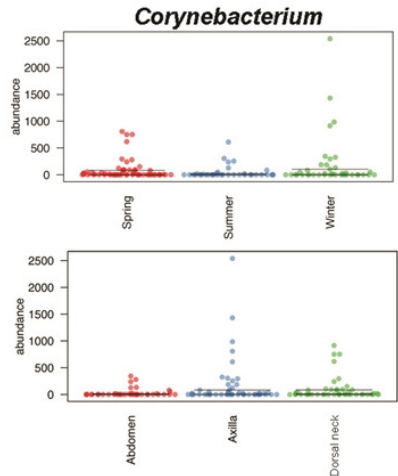

B
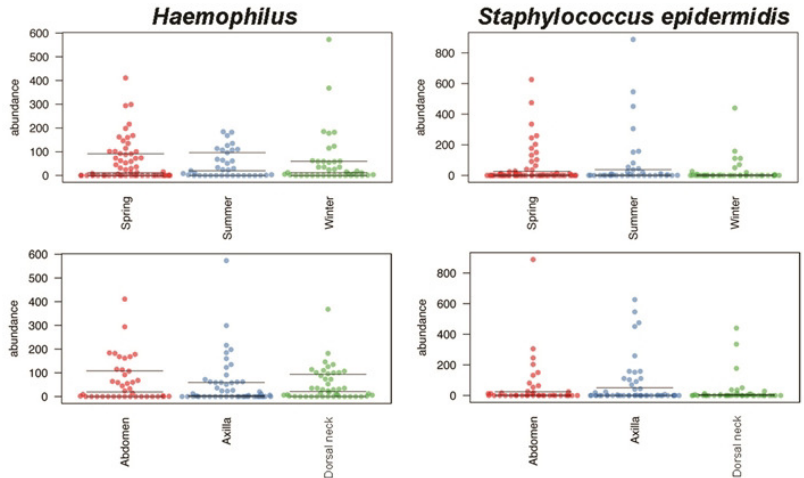

C

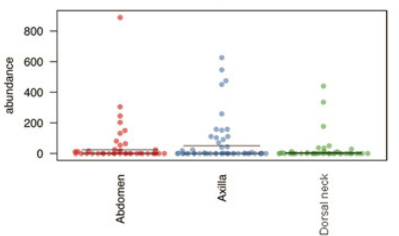

$\mathrm{D}$

Discussion 
280 A wealth of data exists for the bacterial communities inhabiting human skin, but less in known

281 about their counterpart domestic dogs. The purpose of this study was to examine the skin

282 bacterial communities of domestic dogs to assess the effects of cohabitation and season, and to

283 determine if a core skin bacterial community could be identified across a diverse group of

284 animals. The results of these analyses suggest that the canine skin bacterial community is highly

285 diverse and highly variable. Rodrigues-Hoffmann and collaborators came to the same conclusion

286 when examining 12 skin sites from 12 healthy dogs (Rodrigues Hoffmann et al. 2014). They

287 found that Ralstonia was the most abundant genus identified across skin samples, followed by

288 Moraxella and Porphyromonas. In contrast, we identified Propionibacterium acnes as the most

289 abundant OTU, followed by Corynebacterium and Porphyromonas. Interestingly, a study using

290 culture-based methods found $P$. acnes in the epidermis and hair follicles of 7 of $11(63.6 \%)$ dogs

291 suggesting that this bacterium is indeed an important skin resident of dogs (Harvey et al 1993).

292 The differences between this and Hoffman's study are likely a factor of the variability of the

293 canine skin microbiota, since each dominant OTU identified here was indeed highly variable

294 across samples, and/or DNA extraction techniques, primer selection, and PCR parameters. Thus,

295 there is certainly a collection of specific bacterial species that inhabit the skin of dogs that differs

296 from that of humans, but it is likely impacted dramatically by the innate behaviors of the dog

297 compared to humans.

298

299 There were no significant differences in overall bacterial community structure between the three

300 skin sites examined in this study, but there was a significant effect when samples were grouped

301 by timepoint. Again, while statistically significant, the variability between samples of the same

302 timepoint (season) dampened the effect. Actinobacteria appeared to be found at lower relative 
303 abundance in the Summer samplings as compared to the Winter and Spring samplings. It is

304 unclear if this is a meaningful effect, though, as intuitively one would expect Actinobacteria to

305 be at higher abundance in the Summer when dogs are spending more time outdoors since

306 Actinobacteria are ubiquitous in soil and water. It should also be cautioned that only one

307 timepoint per season was assessed. Sampling across multiple years would be required to make

308 definitive statements regarding a true seasonal effect versus a sampling effect.

310 Unifrac distances revealed that there is a significant cohabitation effect on the dog skin bacterial

311 community. That is, dogs that live together have significantly more similar bacterial

312 communities than dogs not living together. Furthermore, samples from the same dog within a

313 household (at different skin sites) amplify this effect. This supports the conclusions that 1) there

314 is significant sharing of bacteria between dogs within the same household, and 2) skin bacterial

315 communities within the same dog across body sites are more similar than non-self samples.

316 Thus, the individual dog appears to have its own unique bacterial community that is consistent

317 across multiple skin sites within the animal. Notably, the effect of cohabitation on the dog skin

318 bacterial communities observed here was less than the increased sharing of microbes between

319 household human partners mediated by household dogs, observed by Song et al. (Song et al.

320 2013). This is expected, though, as the referenced study examined owner hands. Certainly, most

321 dogs within the same household are less likely to have direct intimate contact with each other as

322 compared to the owner-dog interaction.

323

324 Finally, there has been much work aimed at the effects of dog ownership on allergies and asthma

325 in humans, exemplified by a recent study demonstrating that exposure to pets and farm animals 
326 reduces the risk of childhood asthma (Fall et al. 2015). Our data and the results of others indicate

327 that dogs provide a rich source of environmental bacteria to the household, and a study using

328 vacuum settled dust found that dog ownership has also been shown to positively impact the

329 diversity and evenness of bacterial communities in the home (Kettleson et al. 2015). This further

330 indicates the role of the household dog in facilitating the introduction and dissemination of a rich

331 bacterial community throughout the household.

\section{Acknowledgements}

334 Bioinformatics were supported using tools available from the Minnesota Supercomputing 335 Institute. 
336

337

338

339

340

341

342

343

344

345

346

347

348

349

350

351

352

353

354

355

356

357

358

\section{References}

Capone KA, Dowd SE, Stamatas GN, and Nikolovski J. 2011. Diversity of the human skin microbiome early in life. J Invest Dermatol 131:2026-2032.

Caporaso JG, Kuczynski J, Stombaugh J, Bittinger K, Bushman FD, Costello EK, Fierer N, Pena AG, Goodrich JK, Gordon JI, Hutley GA, Kelley ST, Knights D, Koenig JE, Ley RE, Lozupone CA, McDonald D, Muegge BD, Pirrung M, Reeder J, Sevinsky JR, Turnbaugh PJ, Walter WA, Widmann J, Yatsunenko T, Zaneveld J, and Knight R. 2010. QIIME allows analysis of high-throughput community sequencing data. Nat Methods 7:335-336.

Cole JR, Wang Q, Cardenas E, Fish J, Chai B, Farris RJ, Kulam-Syed-Mohideen AS, McGarrell DM, Marsh T, Garrity GM, and Tiedje JM. 2009. The Ribosomal Database Project: improved alignments and new tools for rRNA analysis. Nucleic Acids Res 37:D141-145.

Costello EK, Lauber CL, Hamady M, Fierer N, Gordon JI, and Knight R. 2009. Bacterial community variation in human body habitats across space and time. Science 326:16941697.

Dominguez-Bello MG, Costello EK, Contreras M, Magris M, Hidalgo G, Fierer N, and Knight R. 2010. Delivery mode shapes the acquisition and structure of the initial microbiota across multiple body habitats in newborns. Proc Natl Acad Sci U S A 107:11971-11975.

Edgar RC. 2010. Search and clustering orders of magnitude faster than BLAST. Bioinformatics $26: 2460-2461$.

Fall T, Lundholm C, Ortqvist AK, Fall K, Fang F, Hedhammar A, Kampe O, Ingelsson E, and Almqvist C. 2015. Early exposure to dogs and farm animals and the risk of childhood asthma. JAMA Pediatr 169:e153219. 
359 Flores GE, Caporaso JG, Henley JB, Rideout JR, Domogala D, Chase J, Leff JW, Vazquez-

360 Baeza Y, Gonzalez A, Knight R, Dunn RR, and Fierer N. 2014. Temporal variability is a personalized feature of the human microbiome. Genome Biol 15:531.

362 Grice EA, Kong HH, Conlan S, Deming CB, Davis J, Young AC, Bouffard GG, Blakesley RW, 363 Murray PR, Green ED, Turner ML, and Segre JA. 2009. Topographical and temporal diversity of the human skin microbiome. Science 324:1190-1192.

365 Grice EA, Kong HH, Renaud G, Young AC, Bouffard GG, Blakesley RW, Wolfsberg TG, Turner ML, and Segre JA. 2008. A diversity profile of the human skin microbiota. Genome Res 18:1043-1050.

Grice EA, and Segre JA. 2011. The skin microbiome. Nat Rev Microbiol 9:244-253.

Haas BJ, Gevers D, Earl AM, Feldgarden M, Ward DV, Giannoukos G, Ciulla D, Tabbaa D, Highlander SK, Sodergren E, Methe B, DeSantis TZ, Petrosino JF, Knight R, and Birren BW. 2011. Chimeric 16S rRNA sequence formation and detection in Sanger and 454pyrosequenced PCR amplicons. Genome Res 21:494-504.

Harvey RG, Noble WC and Lloyd DH. 1993. Distribution of propionibacteria on dogs: A preliminary report of the findings on 11 dogs. $J$ Sm Anim Pract 34: 80-84.

Kettleson EM, Adhikari A, Vesper S, Coombs K, Indugula R, and Reponen T. 2015. Key determinants of the fungal and bacterial microbiomes in homes. Environ Res 138:130135.

Li K, Bihan M, and Methe BA. 2013. Analysis of the stability and core taxonomic memberships of the human microbiome. PLoS ONE 8:e63139.

Lozupone C, and Knight R. 2005. UniFrac: a new phylogenetic method for comparing microbial communities. Appl Environ Microbiol 71:8228-8235. 
382 Oh J, Byrd AL, Park M, Kong HH, and Segre JA. 2016. Temporal Stability of the Human Skin 383 Microbiome. Cell 165:854-866.

384 Oh J, Conlan S, Polley EC, Segre JA, and Kong HH. 2012. Shifts in human skin and nares microbiota of healthy children and adults. Genome Med 4:77.

386 Rodrigues Hoffmann A, Patterson AP, Diesel A, Lawhon SD, Ly HJ, Elkins Stephenson C, Mansell J, Steiner JM, Dowd SE, Olivry T, and Suchodolski JS. 2014. The skin microbiome in healthy and allergic dogs. PLoS One 9:e83197.

389 Song SJ, Lauber C, Costello EK, Lozupone CA, Humphrey G, Berg-Lyons D, Caporaso JG, Knights D, Clemente JC, Nakielny S, Gordon JI, Fierer N, and Knight R. 2013. 2:e00458. 


\section{Figure Legends.}

395

396

397

398

399

400

401

402

403

404

405

406

407

408

409

410

411

412

413

414

415

416

417

418

Figure 1. Individual samples classified by Greengenes according to bacterial class, grouped by skin site, and ordered by sample number. The top chart is Spring samples, Middle chart is Summer samples, and bottom chart is Winter samples.

Figure 2. Beeswarm plots of Shannon diversity values with median and interquartile ranges for samples grouped by season (A) and skin site (B).

Figure 3. Principle coordinate analysis (PCoA) plots of individual samples. On the left, samples are colored by skin site using unweighted matrices $(A)$ or weighted matrices $(\mathrm{C})$. On the right, samples are colored by season site using unweighted matrices (B) or weighted matrices (D).

Figure 4. Comparison of unweighted and weighted Unifrac distance matrices between samples when grouped by a variety of different criteria. "Same" indicates average dissimilarity between samples within the same grouping, whereas "different" indicates average dissimilarity between samples between different groupings. Panel A uses unweighted Unifrac distances and Panel B uses weighted Unifrac distances. Those comparisons that are statistically significant $(\mathrm{P}<0.05)$ are indicated with asterisks. Error bars represent 95\% confidence intervals.

Figure 5. OTUs present in $>50 \%$ of all samples by group. Panel A depicts samples by skin site, and Panel B depicts samples by season.

Figure 6. Distribution of selected OTUs identified and classified using the Greengenes database. Beeswarm plots depict individual sample OTU abundance based on 5,000 normalized reads per 
419 sample. Boxplots indicate median and quartile ranges for each OTU. The top plot for each OTU

420 categorizes samples by season, whereas the bottom plots categorize samples by skin site. Panel A 421 = Propionibacterium acnes, Panel B = Corynebacterium, Panel C = Haemophilus, and Panel D = 422 Staphylococcus epidermidis.

423

424

425 